\title{
Diskurs und Hegemonie - Deutungsstrategien in der Frankfurter Stadtentwicklungspolitik
}

\author{
«Die Macht kommt von unten, d.h., sie beruht nicht auf der allgemeinen Matrix einer globalen Zweitei- \\ lung, die Beherrscher und Beherrschte einander entgegensetzt» (FOUCAULT 1983: 115).
}

\begin{abstract}
Wir möchten in diesem Aufsatz Anwendungsmöglichkeiten diskursanalytischer Verfahren in der Geographie aufzeigen. An einem Beispiel aus der Frankfurter Stadtentwicklungspolitik diskutieren wir, wie es sozialen Gruppen gelingt, ihre Aneignungsvorstellungen gesellschaftlicher Räume als verbindliches Konzept zu verallgemeinern. Bei der Diskursanalyse geht man davon aus, daß das theoretische Wissen der Akteure von den sozialen Verhältnissen, in denen sie leben, handlungsanleitend ist. Die Analyse von Diskursen kann deshalb einen wichtigen Beitrag zur Untersuchung der Herausbildung historisch-konkreter sozialer Verhältnisse leisten. Damit bietet sie auch Anknüpfungspunkte zu kritischen gesellschaftstheoretischen Ansätzen wie etwa dem auch in der deutschsprachigen Geographie rezipierten Regulationsansatz (OSSENBRÜGGE 1996).

Zum besseren Verständnis unseres diskursanalytischen Vorgehens klären wir im folgenden zuerst die Begriffe Ideologie, Macht, Hegemonie und Diskurs sowie die theoretischen Verbindungen, die zwischen ihnen bestehen. Danach geben wir anhand der Frankfurter Grüngürtelplanung ein empirisches Beispiel diskursanalytischen Vorgehens.
\end{abstract}

\section{Ideologie und Wirklichkeit}

Voraussetzung für ein Verständnis der Diskursanalyse und des Begriffes «Diskurs» ist, daß Ideologie nicht als «Ensemble von gleichsam luftigen Ideen» (SABLOWSKI 1994: 146) bzw. als «falsches Bewußtsein» oder als verkehrte Abbildung von Wirklichkeit verstanden werden kann. Wesentliche Anstöße für ein anderes Verständnis von Ideologie kamen vor allem von ALTHUSSER (1977) und FOUCAULT $(1978 ; 1983 ; 1991)$. Zum einen zeigte FOUCAULT, daß die Entgegensetzung von Wahrheit und Ideologie, die der traditionelle Ideologiebegriff impliziert, verhindert, Wahrheit selbst zum Gegenstand ideologietheoretischer Analysen zu machen (z. B. FOUCAULT 1991). Zum anderen hoben beide Autoren den produktiven, Handlungen und damit Wirklichkeit generierenden Charakter von Ideologien hervor und betonten damit die Materialität von Ideologien. Ideologien sind nicht Ausdruck der Wirklichkeit, umgekehrt, sie bringen Wirklichkeit hervor. Individuen werden sich mittels
Ideologien über die Verhältnisse, in denen sie leben, klar. Dabei greifen sie auf jeweils vorgefundene, historisch gewordene Erklärungsmuster zurück, die Handlungen und Ereignissen Sinn geben. Diese Sinngebung findet innerhalb gesellschaftlicher Institutionen, Regeln und Rituale statt. Der Sinngebungsprozeß wird Diskurs genannt. Erst in der Praxis der Diskurse erhalten Gegenstände und Zeichen einen Sinn, sie haben also keine Bedeutung «an sich». Diese Sinngebung ist handlungsanleitend für Individuen und generiert insofern Wirklichkeit. Wenn sich Individuen mittels der Diskurse die Welt erklären und entsprechend handeln, dann ist es für Handlungen nicht unerheblich, wie soziale Praktiken sprachlich begründet werden. $\mathrm{Ob}$ beispielsweise der Frankfurter Grüngürtel als Naturschutzgebiet im Sinne eines Gegensatzes von Natur und Mensch oder als Produkt gesellschaftlichen Umgangs mit Natur begriffen wird, legt ganz unterschiedliche Verhaltensweisen nahe und beeinflußt die Gestaltung eines solchen Raumes.

An dieser Stelle wird der FOUCAULTsche Begriff der Macht wichtig. Macht kann zunächst als Befähigung bzw. Beschränkung eines Individuums in seinen Handlungsmöglichkeiten definiert werden. Diskurse befähigen oder beschränken das Handeln von Individuen, indem sie Deutungsmuster zur Verfügung stellen und dadurch Macht ausüben (JÄGER 1993: 152, LINK 1986: 71). Um dies zu verstehen, muß der Machtbegriff von seiner herkömmlichen negativen Vorstellung als Gesetz, Verbot, Repression gelöst werden. Macht im Sinne FOUCAULTs ist nicht funktional im Sinne bestimmter Praktiken zur Aufrechterhaltung eines Herrschaftsverhältnisses einer einheitlichen, zentralen Macht über die Masse der Beherrschten zu verstehen. Mit Macht ist zunächst eine «komplexe strategische Situation in einer Gesellschaft» gemeint (FOUCAULT 1983: 114). Gesetze bzw. staatliche Institutionen, die gemeinhin als Machtinstrumente gelten, sind nach FOUCAULT eher als «Kristallisierung» oder «Endform» «unaufhörlicher Kämpfe» und «Strategien» zu verstehen (ebd.).

Wilfried Körner, Institut für Kulturgeographie, Stadt- und Regionalforschung, J.-W.-Goethe-Universität, Frankfurt am Main;

Christoph Pilgrim, J.-W.-Goethe-Universität, Frankfurt am Main 
Entsprechend diesem Machtbegriff dient die sinnstiftende Praxis der Diskurse weder einer zentralen Macht, noch ist grundsätzlich vorbestimmt, wem welcher Diskurs nützt. Vielmehr können Diskurse «gleichzeitig Machtinstrument und -effekt sein, aber auch Hindernis, Gegenlager, Widerstandspunkt und Ausgangspunkt für eine entgegengesetzte Strategie» (FOUCAULT 1983: 122). Diskurse stabilisieren, destabilisieren oder modifizieren soziale Verhältnisse. Damit öffnet Foucault der Gesellschaftsanalyse ein weites Untersuchungsfeld das z. B. von den Deutungsmustern der Wissenschaften bis hin zum Alltagswissen breiter Bevölkerungsgruppen reicht. Mit seinem Machtbegriff wird es möglich, Argumentationsmuster, die auf "Tatsachen» bzw. "Wahrheiten» oder auf Selbstverständlichkeiten des Alltags beruhen, als Diskurse zu analysieren, die sich nicht als «besseres Argument» durchsetzen, sondern als wirksame strategische Elemente im Kampffeld der gesellschaftlichen Kräfteverhältnisse (FOUCAULT 1978: 53 und 1983: 123). Die Sprachwissenschafter LINK und JÄGER weisen in ihren Analysen des Alltagswissens mehrfach auf eines jener strategischen Elemente hin, das in besonderer Weise machtwirksam sein kann. Die Macht des Alltagswissens eines kulturellen Zusammenhangs speist sich unter anderem aus einem synchronen System kollektiver Symbole (LINK 1982: 11); Kollektivsymbole deshalb, weil sie vielen Menschen - ohne daß sie näher erklärt werden müßten - unmittelbar einleuchten (vgl. JÄGER 1993: 161) und daher keiner weiteren Erklärung oder Deutung bedürften - sie sind eben «wahr». Die Stärke dieser Symbolik liegt darin, daß sie den Individuen komplexe Sachverhalte anschaulich macht und damit die Individuen zu Handlungen befähigt oder einschränkt. Bekannt wurden JÄGERs und LINKs Analysen zu den Diskursen um Asylbewerber und Flüchtlinge, in denen stark mit Naturkatastrophenmetaphern gearbeitet wird (Stichwort «Asylantenflut»). In unserem Beispiel der Frankfurter Grüngürtelplanung werden wir zeigen, wie ein bestimmter Begriff von Natur als Kollektivsymbol und Handlungsanleitung fungiert und somit gesellschaftliche Kräfteverhältnisse beeinflußt.

Wenn Diskurse soziale Verhältnisse sowohl stabilisieren als auch destabilisieren können, dienen sie also nicht einfach der Legitimation der Herrschaft einer zentralen Macht. Bedeutet dies aber, daß alle Individuen gleichermaßen an dem Kampf um Bedeutungen und Sinngebung beteiligt sind?

Um diese Frage zu beantworten, muß ein weiterer Begriff eingeführt werden, der der Hegemonie. Herrschaft wird nicht durch Legitimation aufrechterhalten, eher wird durch einen Komplex praktischer und theoretischer Tätigkeiten ein «tätiger, aktiver Konsens» der Regierten organisiert (GRAMSCI 1967: 354). Die Organisierung dieses Konsenses findet in der Sphäre des erweiterten bürgerlichen Staates, der «Zivilgesellschaft», statt (GRAMSCI 1991). Hier werden kollektive Lebensformen diskursiv ausgebildet und verallgemeinert. Diese diskursive Produktion muß jedoch als besonderes soziales Verhältnis analysiert werden, weil sie selbst den kapitalistischen Bedingungen der Trennung von Kopfund Handarbeit unterworfen ist (SABLOWSKI 1994: 209). Die Bedeutungskonstruktionen sind in unserer Gesellschaft in erster Linie das Metier professionalisierter Intellektueller. Diese überformen soziale Praktiken diskursiv, systematisieren sie und stabilisieren sie damit. «Intellektuelle» sind in diesem Zusammenhang nicht die alltagssprachlich so bezeichneten Berufsgruppen, sondern - nach GRAMSCI (1980) - aufgrund ihrer Stellung in den gesellschaftlichen Verhältnissen als Führer und Organisatoren gesellschaftlicher Kollektive zu verstehen. Die Fähigkeit einer Klasse oder eines Bündnisses, ihre Interessen als Interessen einer ganzen Gesellschaft darzustellen sowie den realen Prozeß der Verallgemeinerung von Interessen in einem stets prekären Gleichgewicht, nennen wir mit GRAMSCI Hegemonie.

\section{Das Projekt Grüngürtel Frankfurt am Main}

Das Grüngürtelprojekt der Stadt Frankfurt wurde 1990 durch den neugebildeten rot-grünen Magistrat initiiert. Wichtigstes Ziel war die langfristige Sicherung und Entwicklung der im äußeren Bereich der Stadt gelegenen Freiflächen - insgesamt knapp ein Drittel des Stadtgebietes. Dieses «Schlüsselelement der sozialen und ökologischen Politik des Magistrats» (STADT FRANKFURT AM MAIN 1991: 238) war darüber hinaus als umfassendes Stadtentwicklungsvorhaben mit weitreichenden ökologischen und sozialen Zielsetzungen konzipiert (KOENIGS 1991: 238 ff., LIESER 1991: 11 ff.). Alle Bevölkerungsgruppen sollten an der Planung und Nutzung des Grüngürtels beteiligt werden (STADT FRANKFURT AM MAIN 1991: 34). Die Projektierung bezog sich zunächst auf einen Zeitraum von 10 Jahren mit einem Investitionsvolumen von 300 Mio. DM. Nach vorangegangenen Kürzungen ist im städtischen Doppelhaushalt 1998/99 die Finanzierung des Projektes aber bereits vollständig eingestellt worden (FRANKFURTER RUNDSCHAU vom 13.1.98), so daß insgesamt nur etwa 15 Mio. DM investiert worden sind (LIESER 1996: 236).

Aus machttheoretischer Sicht stellt sich bezüglich der Grüngürtelplanung v. a. die Frage, welche Positionen die verschiedenen sozialen Gruppen im Prozeß der gesellschaftlichen Aneignung des Grüngürtels einnehmen und wie es bestimmten Gruppen gelingt, ihre Aneignungsvorstellungen als verbindliches Konzept zu verallgemeinern, d.h. also hegemoniale Verhältnisse herzustellen. Diese Fragestellung wurde mittels diskursanalytischer Instrumentarien am Beispiel des Stadtteils Sossenheim untersucht. Frankfurt-Sossenheim war innerhalb der Grüngürtelplanung ein Bereich «oberster Priorität» (KOENIGS 1993: 40). Das Sossenheimer Unterfeld gehört zu den größten zusammenhängenden Flächen des Grüngürtels, bestehend aus landwirtschaftlichen Flächen, Dauerkleingärten, Freizeitgärten, Streuobst- und Grünlandbeständen. Hier wurde 1992 ein Landschaftsplan von einem externen Planungsbüro er- 
stellt, der vom Umweltamt betreut wurde, planungsrechtlich allerdings nicht verankert ist. Die rechtliche Sicherung der Grüngürtelfläche erfolgte zunächst durch die "Grüngürtelcharta» (KOENIGS 1991: 238) und im weiteren durch die Ausweisung in Landschaftsschutzzonen sowie die Einbeziehung in die Bauleit- und Regionalplanung.

\section{Untersuchung und Methodik}

Zur Hoch-Zeit der Planungsbestrebungen wurde 1994 zum Thema "Grüngürtel in Sossenheim» eine diskursanalytisch orientierte Untersuchung durchgeführt (KÖRNER 1998). Hierzu wurden 19 Interviews mit städtischen und Sossenheimer «Schlüsselpersonen» geführt, hinzu kamen die Teilnahme an einer Sitzung des örtlichen Vereinsrings, Präsentationen des Grüngürtelprojektes vor Ort und informelle Gespräche im Umfeld der Planung. Zudem lag eine Vielzahl von schriftlichen Aussagen im Rahmen der Grüngürtelplanung vor.

Es handelte sich bei den Interviews um leitfadengestützte Gespräche, die die subjektive Wahrnehmung und Be-Deutung des Grüngürtels, aber auch die Wahrnehmung von Kommunikationsstrukturen zum Inhalt hatten. In einer gezielten qualitativen Stichprobe wurden PlanungsspezialistInnen aus unterschiedlichen Bereichen, LokalpolitikerInnen und VertreterInnen lokaler Vereine und Initiativen sowie lokale «Gewährsleute» ausgewählt. Die Auswahl der InterviewpartnerInnen erfolgte aufgrund der Position an kommunikativen Schnittstellen. Diese Personen sind wegen ihrer Position wesentlich an der Produktion bzw. Verbreitung der Diskurse beteiligt. Die relativ geringe Zahl von Interviews ist vertretbar, da in diskursanalytischen Untersuchungen davon ausgegangen wird, daß die Zahl von Aussagen zu einem Themenkomplex endlich ist. Weil Diskurse soziale Aushandlungsprozesse und Konsensbildungen spiegeln, gibt es nur eine beschränkte Zahl von gesellschaftlich denkbaren Varianten. Die relative Vollständigkeit eines Diskursstranges wird dann dadurch erreicht, indem die Materialsammlung fortgesetzt wird, bis keine neuen Elemente hinzukommen (JÄGER 1993: 207). JÄGER weist nach, daß bei einer Analyse von 22 Interviews zum Thema «alltäglicher Rassismus» nach der Hälfte der Interviews «keine neuen Tatsachen zu Tage kamen» (JÄGER 1993: 217).

Alle Interviews wurden vollständig transkribiert und in mehreren Stufen verdichtet und ausgewertet. Zunächst wurden Sinnabschnitte in ihren Hauptaussagen zusammengefaßt, anschließend diese Zusammenfassungen mit Überschriften versehen, so daß von jedem Interview schließlich eine überschaubare Struktur der Aussagen vorlag. Außerdem wurden Besonderheiten zu jedem Interview speziell vermerkt. Nachdem die individuellen Relevanzstrukturen herausgearbeitet waren, wurden diese in einem weiteren Schritt zu konsistenten Diskursen verbunden, um gesellschaftlich relevante, handlungsbezogene Deutungsmuster zu ermitteln und auf diese Weise den «Machtgehalt» der sprachlichen Äußerungen deutlich zu machen.

Im folgenden stellen wir nun die Ergebnisse der Analysen vor. Hegemonisierungsprozesse in der Grüngürtelplanung werden zunächst anhand sozialer Positionierungen in institutionellen und netzwerkartigen Strukturen auf der Stadtteil- und auf der Planungsebene dargestellt. Im Anschluß daran werden die spezifischen Ausprägungen von Diskurssträngen in diesen Institutionen und Netzwerken untersucht.

\section{Kontextualisierung der Diskurse: Institutionen und Netzwerke}

Aussagen über die Handlungsmöglichkeiten der Akteure und deren Einflußmöglichkeiten in Diskursen können über die Kontextualisierung der Aussagen der Sprechenden gemacht werden. Hierzu erscheint es uns sinnvoll, die Position der Sprechenden in Netzwerken und Institutionen sowie die Beziehungen unterschiedlicher Institutionen und Netzwerken zueinander näher zu untersuchen. Mit Netzwerken und Institutionen sind hier gesellschaftlich bedeutsame Kommunikationsstrukturen mit strategischer Ausrichtung gemeint. Institutionen werden verstanden als Kommunikationsstrukturen, die rechtlich fixierte, in der Regel hierarchisch organisierte Entscheidungswege aufweisen. Netzwerke sind nichtinstitutionalisierte, kommunikative Verflechtungen, durch die ebenfalls gemeinsame Ziele der Akteure verfolgt werden. Um Einflußmöglichkeiten zu realisieren, können Netzwerke und Institutionen Verbindungen zu anderen Strukturen diese Art aufbauen und nutzen (vgl. SCHWEIZER 1988: $16 \mathrm{ff}$., HAMM/NEUMANN 1996). Netzwerke benötigen in der Regel einen Zugriff auf institutionelle Machtressourcen.

\section{Institutionen und Netzwerke auf Stadtteilebene}

Die städtebauliche Trennung der wesentlichen Siedlungsteile Sossenheims in Alt-Sossenheim und vier Großsiedlungen des sozialen Wohnungsbaus spiegelt sich in den örtlichen Kommunikationsstrukturen wider. Der dörfliche Ortskern Sossenheims wird von einem engen kommunikativen Flechtwerk umspannt, das sich aus persönlichen, familiären, aber auch institutionalisierten Zusammenhängen des Vereinslebens ergibt. Bezüge zu den Siedlungen des sozialen Wohnungsbaus bestehen nur punktuell, in Gesprächen mit AltSossenheimer BürgerInnen werden diese Siedlungen als «Fremdkörper» bezeichnet.

Strategische Bedeutung hat hier eine lokale Metastruktur, die aus Vereinsvorständen, dem örtlichen Vereinsring als «Verein der Vereine», einer «Interessengemeinschaft Sossenheimer Gewerbetreibender» und dem «Sossenheimer Wochenblatt» besteht. Übereinstimmend berichten alle Befragten, da $ß$ diese Institutionen eindeutig von BewohnerInnen aus dem dörflichen Kern, 
häufig alteingesessenen Familien des Stadtteils, dominiert werden. Diese Institutionen sind personell sehr eng miteinander verbunden - einige wenige Personen besetzen die wesentlichen Positionen in diesem Netzwerk. Von besonderer strategischer Bedeutung ist das «Sossenheimer Wochenblatt». Es wird an alle örtlichen Haushalte verteilt und hat damit einen weitreichenden kommunikativen Zugriff auf die Bevölkerung. Laut eigener Einschätzung verfügen einzelne Personen dieser Netzwerkstruktur über gute Verbindungen zur Politik. In der Tat konnte der Vereinsring bei allen größeren Neubauprojekten der jüngsten Zeit seinen Einfluß geltend machen. Somit kommt diesem Netzwerk auch überlokale Bedeutung zu.

In den Siedlungen des sozialen Wohnungsbaus sind ähnliche Strukturen nur rudimentär vorhanden. Einzig der Mieterverein der Henri-Dunant-Siedlung, einer eher kleinbürgerlich strukturierten Siedlung der 60er Jahre, weist solche strategischen Elemente auf. Dieser Verein hat sich 1985 aus einem Mieterbeirat im Zusammenhang mit dem Verkauf der Siedlung gebildet. Er beschäftigt sich vorwiegend mit Problemen, die die Siedlung direkt betreffen, und verfügt nicht über die Bedeutung des Alt-Sossenheimer Netzwerks.

Die strategische Bedeutung der einzelnen sozialen Gruppen in Sossenheim kommt u.a. in deren Präsenz in der Lokalberichterstattung zum Ausdruck. Eine Durchsicht der FRANKFURTER RUNDSCHAU zwischen 1988 und 1993 ergab, daß 29 Artikel Themen behandelten, die vorrangig Alt-Sossenheim betrafen und lediglich $18 \mathrm{mal}$ Themen aus den Siedlungen aufgegriffen wurden.

Eine weitere wichtige soziale Trennlinie innerhalb Sossenheims verläuft zwischen den Bevölkerungsgruppen mit und ohne deutschen Paß. MigrantInnen verfügen im Stadtteil über keine eigene Interessenvertretung, auch spezielle institutionalisierte Kommunikationstreffpunkte fehlen nahezu vollständig. MigrantInnen sind laut Angabe des Vereinsrings nur zu einem geringen Teil Mitglieder in den örtlichen Vereinen. Diese Trennung wird besonders durch Abgrenzungsstrategien der deutschen Mehrheitsbevölkerung zur Hochhaussiedlung «Robert-Dissmann» deutlich, die als «sozialer Brennpunkt» mit hohem AusländerInnenanteil stigmatisiert wird. Diese Siedlung wird von deutschen SossenheimerInnen aufgrund der spezifischen Anordnung der Baukörper gerne mit dem mythischen Begriff «Tatzelwurm» (= sagenhaftes Ungeheuer) belegt.

\section{Institutionen und Netzwerke auf der Planungsebene}

An der Planung in Sossenheim waren unterschiedliche Institutionen beteiligt. Zu Beginn des Projektes 1990 wurde die gesamte konzeptionelle Planung und Koordinierung von dem externen «Projektbüro Grüngürtel» übernommen. Die konkrete Landschaftsplanung für das Sossenheimer Unterfeld wurde dann von einem privaten Planungsbüro erarbeitet und vom Umweltamt betreut. Für die Umsetzung des Planes war die Grüngür-
tel-GmbH zuständig, die 1992 als städtische Gesellschaft gegründet wurde und sich personell teilweise aus dem Projektbüro rekrutierte. Die Grüngürtel-GmbH wurde bereits 1996 nach Beendigung der rot-grünen Koalition unter dem Druck der zunehmenden Finanzkrise wieder aufgelöst.

Die für die langfristige Umsetzung der Planung zuständige Grüngürtel-GmbH verfügte aufgrund unklarer institutioneller Einbindung und Kompetenz über ein beschränktes strategisches Potential. Einerseits hatte sie eine gewisse Autonomie außerhalb der Verwaltungsstrukturen, andererseits war sie aber von städtischen Ämtern abhängig, da Investitionen über die einzelnen Haushalte getätigt wurden. Die $\mathrm{GmbH}$ verfügte über keine eigenen Realisierungsmittel. Einer der beiden Geschäftsführer der Grüngürtel-GmbH äußerte sich folgendermaßen: «Unsere Vorstellungen wären in die andere Richtung gegangen, zu sagen, die $\mathrm{GmbH}$ bekommt eine bestimmte Summe, die sie im Grüngürtel investieren kann, insgesamt und verteilt das dann an die einzelnen Ämter, sofern sie selbst bauen bzw. Verträge vergeben. Aber das sind innerhalb der Stadt natürlich starke Auseinandersetzungen, und so stark wollte man die $\mathrm{GmbH}$ zunächst nicht.»

Kommunikation wurde über Arbeitsgruppen oder spezielle Treffen hergestellt, in denen die $\mathrm{GmbH}$ durch die Vermittlung zwischen den jeweils betroffenen Ämtern sowie zwischen städtischen und Stadtteilakteuren Handlungsmöglichkeiten realisieren konnte. Kontakte der Planungsinstitutionen in Sossenheim waren relativ schwach ausgeprägt. Weder Umweltamt noch Planungsbüro waren hier dauerhaft präsent. Die Grüngürtel-GmbH bzw. das Projektbüro hatte Kontakte über den Ortsbeirat (Stadtteilparlament), über einen Volkshochschulkurs und durch mehrere Präsentationen der Planungen vor Ort aufgenommen.

Im Zuge der Befragung wurde deutlich, daß der Ortsbeirat selbst über eine relativ schwache Verankerung im Stadtteil verfügte. Ursache hierfür war der ausgedehnte Zuständigkeitsbereich des Ortsbeirates, der außer Sossenheim noch sieben weitere Ortsteile umfaßt, aber auch die insgesamt zurückgehende Bedeutung der Parteien im Stadtteil. Zitat einer Sossenheimerin: «Die Parteien sind so zurückgegangen in ihrer Substanz. Das sind so wenige Leute, sowohl bei der SPD als auch bei der CDU.» Auch die geringe Wahlbeteiligung bei der Kommunalwahl 1993 von 66,5\% (minus 8,6 Prozentpunkte gegenüber 1989) zeigt dies deutlich.

Die öffentlichen Veranstaltungen wie auch der Volkshochschulkurs wurden eindeutig sowohl personell als auch thematisch von Alt-SossenheimerInnen dominiert. Bezüge der Planungsebene zu MigrantInnen im Stadtteil fehlten vollständig (vgl. auch LANZ 1996: 157, 153). Zusammenfassend kann man hinsichtlich der strategischen Bedeutung von Kommunikations- und Netzwerkstrukturen in Sossenheim sagen, daß komplexe Verflechtungen im dörflichen Kern und die Besetzung zentraler Punkte zu einer relativen Dominanz von alteingesessenen SossenheimerInnen führen. Die Kontakte 
zwischen Planung und Stadtteil waren schwach ausgebildet und bezogen sich in erster Linie auf schlecht funktionierende Strukturen wie den Ortsbeirat oder auf traditionell organisierte Bürgerversammlungen. Bestehende Kontakte bezogen sich v. a. auf Akteure der Alt-Sossenheimer Netzwerkstrukturen. Die Grüngürtel$\mathrm{GmbH}$ als Mittlerin verfügte über eine relativ schwache Position im Gesamtprozeß.

\section{Diskursstrukturen}

Die Grüngürteldiskussion kann in zwei entscheidende Diskursstränge gegliedert werden, die den Gesamtablauf der Planung in Sossenheim maßgeblich beeinflußt haben: den Ökologiediskurs und den Diskurs des Sozialen. Bereits im Projektbüro waren zwei Arbeitsgruppen tätig, die sich mit ökologischen und sozialen Fragen beschäftigten. Diese Diskurse traten nicht auf allen Ebenen der Diskussion auf, zudem war ihre Ausprägung in den verschiedenen Gruppen auch sehr unterschiedlich.

\section{Ökologiediskurs}

Der Ökologiediskurs ist seit Beginn des Grüngürtelvorhabens von dominanter Bedeutung. Dies kommt dadurch zum Ausdruck, daß er auf allen Ebenen in Erscheinung tritt und von allen Beteiligten als wesentlicher Argumentationszusammenhang wahrgenommen wird. Politische Hintergründe wie die Bildung der rotgrünen Koalition und die Zuständigkeit des GRÜNEN Umweltdezernenten für den Grüngürtel anstelle des SPDbesetzten Planungsdezernats verweisen auf eine generelle Zunahme ökologischer Deutungszusammenhänge. In den Veröffentlichungen der Grüngürtelplanung wurde besonderer Wert auf die ökologische Orientierung des Projekts gelegt (vgl. STADT FRANKFURT AM MAIN 1991, KOENIGS 1991). Welches sind die Kennzeichen dieses Diskurses? Und wie stellt sich die Rezeption und Entwicklung dieses Diskurses in den unterschiedlichen Gruppen dar?

\section{Ökologie als Naturschutz}

Die argumentative Konstruktion von Ökologie als Naturschutz ist das am weitesten verbreitete Deutungsmuster des Grüngürtels. Bereits in der Grüngürtelcharta wurde festgeschrieben: «Die ökologischen Werte des Grüngürtels, wie Biotop- und Artenvielfalt, Grundwasserreservoir, Grundwassersysteme und klimatische Potentiale, werden gestärkt und verbessert» (KOENIGS 1991: 238). Die damit verbundenen sprachlichen Darstellungen zielen auf die Konstruktion eines Gegensatzes von Gesellschaft und Natur: Die Natur muß vor dem Menschen geschützt werden. Solche argumentativen Operationen finden sich insbesondere im zuständigen Umweltamt, bei den GRÜNEN im Ortsbeirat, bei der Ortsgruppe des BUND, teilweise auch in der Grüngürtel-GmbH. Am deutlichsten ist diese Position bei dem zuständigen Sachbearbeiter im Umweltamt vertreten, er versteht sich explizit als «Anwalt der Natur». Er formuliert: «... wir nehmen den Naturraumgedanken, den Naturschutzgedanken... also ich setze ihn noch eine Stufe höher an als den Erholungsraum. (...) Und daß nachher Konflikte im sozialen Bereich wie auch immer ausgetragen werden, ich denke, da gibt es wirklich andere Fachleute, die sowas dann sich überlegen müssen. (...) Im Prinzip ist es ein Gegensatz.» Die logische Folge der ideellen Trennung von Gesellschaft und Natur ist eine räumliche Trennung beider Bereiche. So regte das GRÜNE Ortsbeiratsmitglied etwa eine rigorose Trennung von Erholungs- und Naturbereichen an. Er schlägt vor, Aussichtsplattformen im Gelände zu installieren, damit Erholungsuchende in Naturschutzbereiche Einblick bekommen. Der Einblick von oben läßt jeden einzelnen diese Trennung bewußt oder unbewußt nachvollziehen. Diese gewissermaßen räumlich materialisierte Vorstellung verweist in eindrücklicher Form auf die soziale Funktion eines «menschenleeren» Naturbildes als Element der Befriedung sozialer Verhältnisse. So sagt das GRÜNE Ortsbeiratsmitglied weiter: «Ich bin schon der Meinung, daß eine intakte Landschaft und intakte Erholungsflächen rund um einen Stadtteil sich positiv auch auf die soziale Struktur des Stadtteils auswirken können.» Natur wird als nichtsoziales Phänomen zu einem Projekt der Harmonisierung gesellschaftlicher Verhältnisse durch die «Inszenierung einer heilen Welt» (GATHER/UNTERWERNER 1992: 111).

\section{Ökologie und Ethnisierung sozialer Verhältnisse}

Ethnisierung bedeutet, daß Individuen aufgrund ihrer Nationalität oder ethnischen Zugehörigkeit bestimmte Wesensmerkmale zugeschrieben werden und diese zur Erklärung von sozialen oder wirtschaftlichen Phänomenen herangezogen werden (PILGRIM 1998). Manifeste Ethnisierungsstrategien treten besonders im rechten politischen Spektrum auf, um gesellschaftliche Probleme zu thematisieren. Das zugrundeliegende Muster zeigt sich aber auch in anderen sozialen und politischen Gruppen. Die Ergebnisse von Kommunalwahlen zeigen, daß Ethnisierungsdiskurse auf Stadtteilebene relevant sind. Bei den Kommunalwahlen 1993 konnten rechte Gruppierungen in Sossenheim über 20,6\% (gegenüber 12,9\% in Gesamt-Frankfurt) auf sich vereinen (STADT FRANKFURT AM MAIN 1993: 38 ff.) Zwar ergab die Untersuchung der Kommunikationsstrukturen (soweit dies möglich war), daß direkte Kontakte zu rechtsextremistischen Parteien selten sind und auch viele politische Aussagen dieser Gruppierungen nicht gutgeheissen werden. Es finden sich jedoch in einer Vielzahl von Äußerungen zu gesellschaftlichen Phänomenen Deutungsmuster, die Probleme vor Ort mit der Anwesenheit von MigrantInnen in Verbindung bringen, so da $\beta$ hier durchaus von Ethnisierungsdiskursen gesprochen werden kann. Kennzeichnend für dieses Erklärungsmodell ist die summarische Rede von «den Ausländern», die ja faktisch eine äußerst heterogene Gruppe darstellen. 
Ein wichtiges Merkmal des vorgefundenen Ethnisierungsdiskurses besteht darin, kulturelle Andersartigkeit als Ursache für soziale Konflikte im Stadtteil darzustellen und sie als Begründungszusammenhang für eine differente Behandlung von MigrantInnen zu nutzen. Einer der heftigsten Konflikte im Bereich des Sossenheimer Grüngürtels betrifft die am Ortsrand liegenden «Freizeitgärten». Diese Gärten sind planungsrechtlich nicht abgesichert und werden in den Gesprächen häufig als «wilde Gärten» bezeichnet. Übereinstimmend wird angegeben, daß diese Gärten vorwiegend von ausländischen Familien betrieben werden. In fast allen Interviews mit deutschen SossenheimerInnen, besonders mit Alteingesessenen und Mitgliedern des Kleingartenvereins, wird rigoros die Abschaffung dieser Gärten gefordert. Als Ursache sozialer Konflikte mit den BetreiberInnen dieser Gärten wird die kulturelle Andersartigkeit, eine andere Lebens- und Alltagspraxis, genannt. Um nur einige Zitate als Beleg zu nennen: «Dann haben die eine andere kulturelle Einstellung, eine andere Vorstellung von Leben, dann haben die ein anderes Temperament. $\mathrm{Da} ß$ es da dann irgendwelche Konflikte gibt, liegt ja auf der Hand.» «(...) jeder macht das, was er gerade will. Und wenn er am Sonntag Rasen mähen will, dann mäht er am Sonntag Rasen.» «... natürlich eine Minderheit fühlt sich jetzt da vielleicht wohl.»

In einem weiteren argumentativen Schritt kommt es zu einer Verknotung mit dem Ökologiediskurs. In dieser Konstruktion wird kulturelle Andersartigkeit zur Ursache einer fehlenden ökologischen Orientierung von MigrantInnen. Es kommt gewissermaßen zu einer Verschleierung des politischen Ethnisierungsdiskurses durch den «harmloseren» Ökologiediskurs. Ein organisierter Kleingärtner führt dazu aus: «Ich habe viele Bewerber - Jugoslawen oder Türken -, die wollen einen Garten haben, aber die interessiert die Umwelt nicht, die wollen einfach zusammen kommen, die wollen die Familie...» Als ökologische Probleme in den «wilden Gärten» werden Beispiele angeführt, die wenigstens teilweise auch in den gutgeheissenen organisierten Kleingärten auftreten: zunehmende Versiegelung, Nutzung von Stromaggregaten und Rasenmähern oder sanitäre Probleme. So wird denn auch nicht eine «Ökologisierung» der Gärten gefordert, sondern eine völlige Abschaffung der «wilden Gärten». Die Verbindung zum Ökologiediskurs als hegemoniale Argumentationsfigur wird also genutzt, um territoriale Hegemonie zu legitimieren. «Also, ich halte es als Zukunftsaufgabe für eine Großstadt von großer Bedeutung, diese Planung durchzusetzen. Nämlich die bestehende grüne Lunge zu erhalten, und das ist ja wohl auch eigentlich der wahre Inhalt des Grüngürtels (...) Und die geht kaputt z. B. auch durch die scheiß wilden Gärten» (Alt-Sossenheimer). An dieser Stelle ist zu vermerken, daß gegenwärtig entgegen den Vorstellungen des Landschaftsplans Sossenheim, der von dem Erhalt aller Gartengebiete ausging, ein großer Teil der "wilden Gärten» aufgrund der Ausweisung als Landschaftsschutzgebiet aufgelöst wird (FRANKFURTER RUNDSCHAU 22.5.98).

\section{Diskurs des Sozialen}

Mit dem Diskurs des Sozialen ist ein zusammenhängendes Erklärungsmuster gemeint, das gesellschaftliche Verhältnisse in das Zentrum der Diskussion über den Grüngürtel rückt. Dieser Diskurs umfaßt im wesentlichen zwei Aspekte: In analytischer Hinsicht wird Natur als gesellschaftliches Produkt definiert: «Landschaft ist kein von Ewigkeit her gegebenes Ding, sondern das Produkt gesellschaftlichen Umgangs mit der Natur. Als sedimentierte Geschichte und aktuelles Politikum wird sie in ihrer Komplexität aber kaum noch durchschaut» (WORMBS 1978: 203, zitiert nach GRÜNGÜRTEL-PROJEKTBÜRO 1990: 71). Der Diskurs des Sozialen impliziert somit eine sozialwissenschaftlich bestimmte Deutung des Ökologiediskurses und tritt damit konkurrierend zur Naturschutzideologie auf.

Praktisch - also im Zusammenhang mit Handlungsaspekten - wird eine Nutzung und Planung des Grüngürtels durch breite Bevölkerungsschichten gefordert bzw. in Aussicht gestellt. Dieser Diskurs wurde v. a. im Grüngürtel-Projektbüro in der Arbeitsgruppe «Raum und Gesellschaft» geführt (STADT FRANKFURT AM MAIN 1991: 25 ff.) und später in Teilen der Grüngürtel-GmbH aufgenommen. In der Grüngürtelcharta wurde dies unter dem Stichwort «Sozialer Nutzen» festgeschrieben: «Der Grüngürtel ermöglicht gesellschaftliche Teilhabe an seinen Ressourcen.» Oder: «Für die verschiedenartigen Nutzungen werden jeweils spezifische soziale Infrastrukturen bereitgestellt, die umweltverträglich sind, aber auch gesellschaftlichen Anforderungen wie dem Gleichheits- und Freiheitspostulat Rechnung tragen» (KOENIGS 1991: 239). Unter diesem Gesichtspunkt wurden auch durchaus soziale Konfliktsituationen im Grüngürtel analysiert. Dies geschah allerdings auf sehr abstrakter Ebene, konkrete Handlungsimplikationen waren in diesem Diskurs weitgehend nicht eingeschlossen (vgl. KEIL/RONNEBERGER 1991: 204). So erfolgte etwa auch die Kenntnisnahme von den Sossenheimer Machtstrukturen nur spät und unvollständig.

Mit der Absicht, allen Bevölkerungsgruppen Mitgestaltung zu ermöglichen, hätte die Organisation der Diskussion verstärkt ungleiche Diskursivierungsmöglichkeiten berücksichtigen müssen. Die fehlende Sensibilität für Machtverhältnisse mag ein Grund dafür sein, daß dieser Diskurs des Sozialen im Stadtteil nicht aufgegriffen wurde. Ein weiterer Grund ist darin zu sehen, da $\beta$ der Diskurs auch im Planungsprozeß keine annähernd hegemoniale Bedeutung erlangen konnte im Vergleich zum Naturschutz-Ökologiediskurs, der sowohl auf der Planungsebene, aber auch in spezifischer Reformulierung in Sossenheim aufgegriffen wurde. Schließlich trug die relativ schwache Position der Grüngürtel-GmbH dazu bei, daß ein Diskurs des Sozialen sich nicht wirklich durchsetzen konnte. 


\section{Zusammenfassung}

Diskursforschung bietet eine wichtige Möglichkeit der Konkretisierung und Operationalisierung der Analyse sozialer Verhältnisse durch die Beschreibung gesellschaftlicher Prozesse der Machtbildung, der Hegemonisierung und der Steuerung von Raumaneignungsprozessen. Die Analyse der Diskurs- und Kommunikationsstrukturen im Frankfurter Grüngürtelprojekt hat deutlich gemacht, daß Diskurse als komplexe Deutungssysteme zur Machtbildung in bestimmten Räumen entscheidend beitragen. Die Realisierung von Machtpotentialen geschieht dabei über Kommunikationskanäle - Institutionen und Netzwerke -, in denen diskursive Inhalte institutionalisiert und transportiert werden. Lokale Diskursformationen funktionieren im Falle Sossenheims über die Reformulierung gesamtgesellschaftlicher bzw. städtischer Deutungsinhalte, deren hegemonialer Charakter genutzt wird. Hier wurde z.B. der Ökologiediskurs in seiner Ausprägung als Naturschutzdiskurs zur Unterstützung des Ethnisierungsdiskurses eingesetzt.

Dies war insbesondere auch deshalb möglich, weil eine Diskurskoalition aus Umweltamt, Umweltverband, Lokalpolitikern und der deutschen Sossenheimer Bevölkerung «Naturschutz» als hegemoniales Deutungsmuster etablierte. Aufgrund der Ausblendung des Sozialen in dieser Naturschutzideologie ist eine Verkopplung mit Ethnisierungsdiskursen überhaupt möglich. Die an Partizipation orientierte Planung konnte die selektive Privilegierung einzelner Bevölkerungsteile wegen einer strukturell schlechten Position, fehlender Handlungsimplikationen des Sozialdiskurses und aufgrund der einseitigen Bezugnahme auf die im Feld lokaler Machtstrukturen bereits dominierenden Gruppen nicht verhindern.

\section{Literatur}

ALTHUSSER, L. (1977): Ideologie und ideologische Staatsapparate. Hamburg.

FOUCAULT, M. (1978): Dispositive der Macht. Berlin.

FOUCAULT, M. (1983): Der Wille zum Wissen. Sexualität und Wahrheit I. Frankfurt am Main.

FRANKFURTER RUNDSCHAU vom 13.1.98: «Koenigs beklagt Mittelstreichung".

FRANKFURTER RUNDSCHAU vom 22.5.98: «Entsorgungsbetrieb räumt Schutt im Sossenheimer Unterfeld ab".
GATHER, M. / UNTERWERNER, P. (1992): Die grüne Weltstadt? - "Grüne» Politik in Frankfurt am Main. In: Geographische Zeitschrift, H. 2, 106-120.

GRAMSCI, A. (1967): Philosophie der Praxis. Frankfurt am Main.

GRAMSCI, A. (1980): Zu Politik, Geschichte und Kultur. Leipzig.

GRAMSCI, A. (1991): Gefängnishefte. Hamburg.

GRÜNGÜRTEL-PROJEKTBÜRO (1990): Werkbericht 2/1990.

Materialien zur Sommerakademie. Frankfurt.

HAMM, B. / NEUMANN, I. 1996: Siedlungs-, Umwelt- und Planungssoziologie. Opladen.

JÄGER, S. (1993): Kritische Diskursanalyse. Eine Einführung Duisburg.

KEIL, R. / RONNEBERGER, K. (1991): Arkadien postmodern. Stadtlandschaft zwischen Streuobst und Gewerbepark. In: KOENIGS, T. (Hrsg.): Visionen offener Grünräume, Grüngürtel Frankfurt, 196-208. Frankfurt/New York.

KOENIGS, T. (1991) (Hrsg.): Visionen offener Grünräume, Grüngürtel Frankfurt. Frankfurt/New York.

KOENIGS, T. (1993) (Hrsg.): Stadt-Parks. Frankfurt/New York.

KÖRNER, W. (1998): Der Frankfurter Grüngürtel als sozialer Raum - Diskurse, Raumbilder und Netzwerke. Das Beispiel Sossenheim. = Materialien, Institut für Kulturgeographie, Stadt- und Regionalforschung, Bd. 23. Frankfurt.

LANZ, S. (1996): Demokratische Stadtplanung in der Postmoderne. Wahrnehmungsgeographische Studien zur Regionalentwicklung, $H .15$. Oldenburg.

LIESER, P. (1991): Neuland im Stadtland. Stadtentwicklung mit städtischer Natur und offenen Räumen. In: KOENIGS, T. $(\mathrm{Hg}$.$) : Visionen offener Grünräume, Grüngürtel Frankfurt.$ 11-24. Frankfurt/New York.

LIESER, P. (1996): GrünGürtel: Neue Strategie oder letztes Gefecht für Natur in der Stadt? In: WENTZ, M. (Hrsg.): Stadtentwicklung = Die Zukunft des Städtischen, Bd. 9, S. 232238. Frankfurt/New York.

LINK, J. (1982): Kollektivsymbolik und Mediendiskurse. kultuRRevolution, H.1, 6-21.

LINK, J. (1986): Noch einmal: Diskurs. Interdiskurs. Macht. In: kultuRRevolution, H.11, 4-7.

OSSENBRÜGGE, J. (1996): Einführung: Regulationstheorie und Geographie. In: Zeitschrift für Wirtschaftsgeographie, H.1-2, Jg. 40, 2-5.

PILGRIM, C. (1998): Der ethnisierte Raum. Geographie und Historiographie in den Schulbüchern Mexikos und der USA. Unveröff. Dissertation. Universität Frankfurt am Main.

SABLOWSKI, T. (1994): Zum Status des Hegemoniebegriffs in der Regulationstheorie. In: ESSER, J. / GÖRG, C. I HIRSCH, J. (Hrsg.): Politik, Institutionen und Staat. Zur Kritik der Regulationstheorie. 133-156. Hamburg.

SCHWEIZER, T. (Hrsg.) (1988): Netzwerkanalyse. Ethnologische Perspektiven. Berlin.

STADT FRANKFURT AM MAIN, DEZERNAT FÜR UMWELT, ENERGIE UND BRANDSCHUTZ (1991): Ergebnisbericht Grüngürtel Planung 1990/91. Frankfurt.

STADT FRANKFURT AM MAIN, AMT FÜR STATISTIK, WAHLEN UND EINWOHNERWESEN (1993): Kommunalwahlen 1993 in Frankfurt am Main = Frankfurter Wahlanalysen, $H$. 1, Frankfurt am Main.

WORMBS, B. (1978): Über den Umgang mit der Natur: Landschaft zwischen Illusion und Ideal. Frankfurt am Main. 